\title{
Simulating the Unexpected: Challenge-Centric Simulator Scenario Design for Advanced Flight Crew Training
}

\author{
Dominik Niedermeier*, Jan-Philipp Buch ${ }^{\dagger}$ and Umut Durak ${ }^{\ddagger}$ \\ German Aerospace Center (DLR), Braunschweig, 38108, Germany \\ Frederik Mohrmann ${ }^{\S}$ \\ Netherlands Aerospace Centre (NLR), Amsterdam, 1059 CM, The Netherlands
}

In 2013 the International Civil Aviation Organization (ICAO) published a report on the implementation of Evidence-Based Training (EBT). EBT aims at improving the overall capabilities of a pilot trainee in certain competencies such as decision-making, communication, situational awareness and others. This results in a shift in training focus from maneuvers to the training of competencies, which in turn sets different requirements for the desired simulator training scenarios. This paper presents a training scenario design approach being driven by the expected impact on the crew rather than focusing on specific maneuvers or system failures. The so-called "Challenge-Centric" design approach was applied in the EU project Man4Gen. It forms the basis for the development of a scenario design kit that shall facilitate the design of training scenarios for Evidence-based Training.

\section{Nomenclature}

$\begin{array}{ll}\text { ATC } & =\text { Air Traffic Control } \\ \text { ATO } & =\text { Approved Training Organization } \\ \text { ATHR } & =\text { Autothrust } \\ \text { AVES } & =\text { Air Vehicle Simulator } \\ \text { DFCP } & =\text { Desired Flight Crew Performance } \\ \text { DSL } & =\text { Domain Specific Language } \\ \text { ECAM } & =\text { Electronic Centralized Aircraft Monitor } \\ \text { EBT } & =\text { Evidence-based Training } \\ \text { FADEC } & =\text { Full Authority Digital Engine Control } \\ \text { FCOM } & =\text { Flight Crew Operating Manual } \\ \text { FMS } & =\text { Flight Management System } \\ \text { GRACE } & =\text { Generic Research Aircraft Cockpit Environment } \\ \text { IATA } & =\text { International Air Transport Association } \\ \text { ICAO } & =\text { International Civil Aviation Organization } \\ \text { IDLE } & =\text { Idle thrust setting } \\ \text { IFR } & =\text { Instrument Flight Rules } \\ \text { MBT } & =\text { Maneuver-based Training } \\ \text { MCT } & =\text { Maximum Continuous Thrust } \\ \text { MSTC } & =\text { Modeling and Simulation Technical Committee } \\ \text { QRH } & =\text { Quick Reference Handbook } \\ \text { SES } & =\text { System Entity Structure } \\ \text { SSDWG } & =\text { Simulation Scenario Development Working Group } \\ \text { SOP } & =\text { Standard Operating Procedure } \\ \text { THR } & =\text { Thrust } \\ \text { T/O } & =\text { Take-off } \\ & \end{array}$

\footnotetext{
*Research scientist, Flight Dynamics and Simulation, Institute of Flight Systems.

${ }^{\dagger}$ Research scientist, Flight Dynamics and Simulation, Institute of Flight Systems.

${ }^{\ddagger}$ Research scientist, Flight Dynamics and Simulation, Institute of Flight Systems.

${ }^{\S}$ R\&D Engineer, Aerospace Operations, Operator Performance, Training \& Simulation.
} 


\section{Introduction}

\section{A. The Need for Competency-Based Training}

For many decades full flight simulators have proven to be a powerful tool for training flight crews in airline operations. This is true for the training of specific basic flying skills, the handling of particular system failures as well as the training of decision-making and problem solving processes in a complex operationally relevant scenario. In the early days of simulator training, each scenario was intended to train certain anticipated events and failures, often referred to as Maneuver-Based Training (MBT). This training is still necessary to properly prepare flight crews for critical events that must be recognized rapidly. That is why it was set as regulatory standard. However, as aircraft and operations become increasingly complex, recent accidents and incidents are showing that crews are having difficulty with a different type of challenge in the cockpit: managing unexpected, complex and ambiguous situations. Examples of such cases are Qantas 32 [1] and Air Asia 8501 [2].

Maneuver-based Training does not necessarily prepare flight crews well for these new challenges. In addition, the relevancy of certain scenario elements required by the regulators has changed due to the evolution of modern aircraft and their component reliability. This led to the development of Evidence-based Training by the International Air Transport Association (IATA) Training and Qualification Initiative as defined in the ICAO Document 9995 "Manual of Evidence-based Training" [3]. EBT aims at improving the overall capabilities of a pilot trainee in certain competencies such as decision-making, communication, situational awareness and others. This results in a shift in training focus from maneuvers, to the training of competencies, which in turn sets different requirements for the desired simulator training scenarios. Inherent to scenarios which trigger specific competencies is that they are unfamiliar and pose the crew with a novel situation in which they can practice (or demonstrate) their competencies without being able to have memorized the exact solution for that scenario. The major challenge lies in making several scenarios which all trigger the same competencies with different contexts; this causes such scenario design to be a very challenging and time consuming task for Approved Training Organizations (ATO). The challenge in designing such scenarios was tacked within the European Union research project "Manual Operations of 4th Generation Airliners" (Man4Gen). The design of the Man4Gen simulation scenario was clearly focused on the expected effects on the crew, and not on specific maneuvers or events as in maneuver-based training. In addition, the scenario design process had another challenge in that the scenario was supposed to be comparable in two different simulators (different possibilities and features) as well as to be applied to two different aircraft types (Airbus A320 and A330). However, this challenge forced the designers to consider external factors such as the planned route, location (including the timing of failures) and weather. Further comparability between the experimental runs was achieved by scripting the behavior of the simulated radio contacts, such as the Air Traffic Management controllers, the airline dispatcher as well as the airlines maintenance center. Lastly, parts of the experimental briefing were conducted using video replays to standardize between the two experiment locations.

\section{B. Scenario Design in the Man4Gen Project}

Based on recent incidents and accidents, the Man4Gen problem statement served as the starting point for the conducted research: "Despite the substantial and proven safety benefits of automation systems in 3rd and 4th generation aircraft, evidence indicates that when faced with unexpected and challenging situations, pilots sometimes have difficulties in quickly responding to situations which require a rapid transition in their activity from monitors of very reliable systems, to active and authoritative decision-makers exercising manual control of the aircraft." The Man4Gen project goals were to develop and assess different recommendations in the areas of procedures, flight deck design and training, to support crews during unexpected, complex and ambiguous situations. For the development of the recommendations in these three different areas results of an exploratory simulator study were used. Finally, in order to assess crew behavior with such solutions, a second simulator study was performed on two research simulators: the German Aerospace Center (DLR) Air Vehicle Simulator (AVES) and the Netherlands Aerospace Centre (NLR) research simulator Generic Research Aircraft Cockpit Environment (GRACE). Both simulators are full-flight simulators featuring almost complete cockpit replicas of an Airbus A320 (DLR) or an Airbus A330 (NLR). Both include the simulation of all aircraft systems relevant for the Man4Gen studies, autopilot as well as Fly-by-Wire control modes. For the assessment of the recommendations in the fields of procedures and flight deck design a complex and operationally relevant scenario was designed within the project. In order to evaluate the recommended procedures and flight deck modifications properly, a scenario had to be developed which could specifically challenge crews with surprise, complexity and ambiguity, as these three elements were recurring themes in incidents and accidents in fourth generation airliners [4]. 


\section{Scope of the Paper}

This paper uses the example of the scenario designed for the second Man4Gen simulator study to illustrate the complex design task of creating operationally relevant scenarios to trigger desired crew challenges. This challengecentric approach has the additional benefit of more easily identifying the critical steps to recovery which can be used to assess crew performance in these scenarios, or even be linked to the EBT performance indicators. Based on Man4Gen experience, the paper will discuss how such scenarios can be generated more rapidly in the future. It includes standardization of an efficient and effective scenario development and execution process on flight training devices and research simulators. Later, the paper will hook to DLR's recent research initiative that targets developing a scenario toolchain for supporting training organizations. The toolchain shall support and automate the scenario design based on required core challenges (or alternatively competencies) and the scenario distribution to applications on flight simulators, and the later the execution and monitoring of the scenario. The toolchain development is conducted hand in hand with the development of a formal scenario definition language that shall facilitate required automation.

\section{Challenge-Centric Scenario Design Process in Man4Gen}

For the assessment of the recommendations in the fields of procedures and flight deck design a complex and operationally relevant scenario was designed within the Man4Gen project. This section presents the applied challengecentric design process.

In case of maneuver-based training, scenarios including anticipated system failures are defined by the certification authorities based on relevant system failures identified by the aircraft manufacturers. This approach could be designated as a "system-centric approach". In contrast to this the challenge-centric approach used in Man4Gen first focuses on the expected or desired impact on the crew which then lead to the definition of a certain event in the scenario. Depending on the desired effect this event could be a system failure, an environmental disturbance or any other realistic event in airline operations. It is evident that the effect on the crew is not only depended on the event itself but also on its timing and its combination with other events. This has to be considered during the scenario design process.

The challenge-centric design approach is further clarified by Figure 1

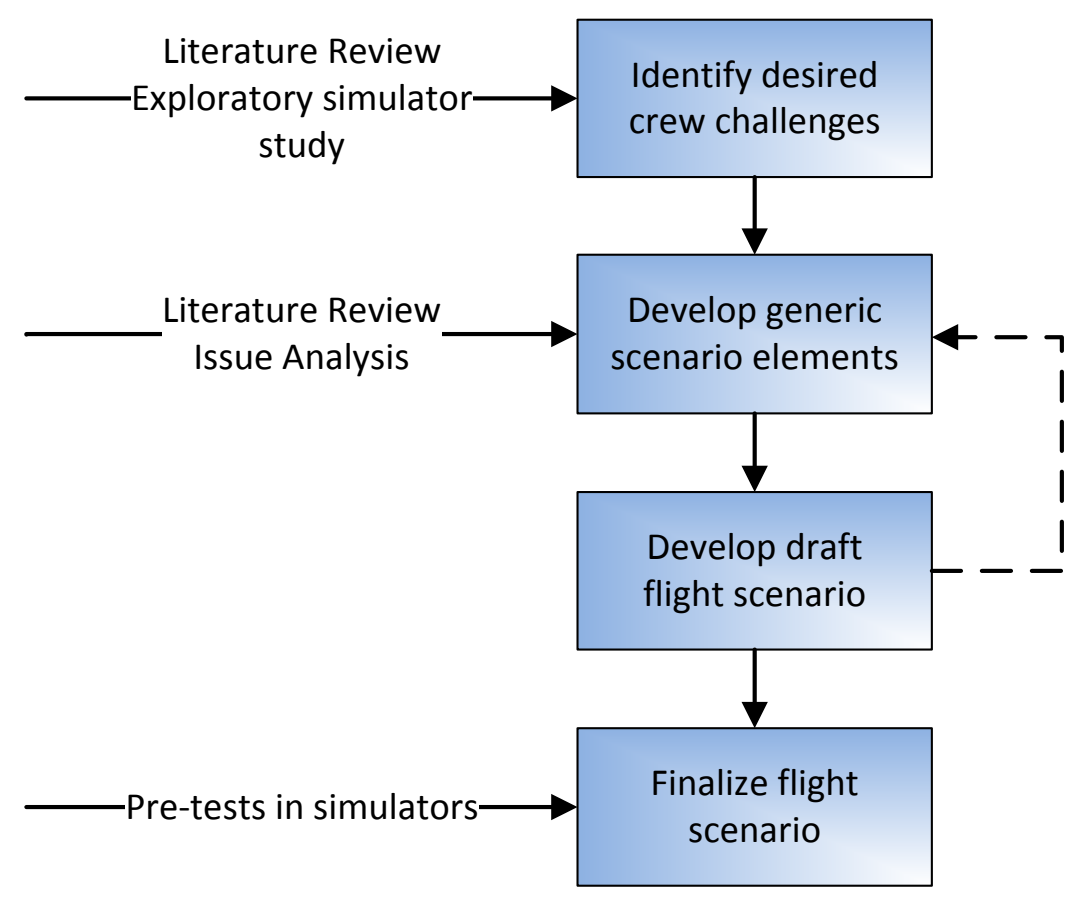

Fig. 1 Challenge-centric scenario design process 


\section{A. Design Constraints}

The Man4Gen scenario of the first (exploratory) simulator study was designed as a quickly deteriorating failure situation with a sudden rise in workload and was mainly focused on the crew's decision to take over manual control. Therefore, besides the crew's situation awareness and their decision-making processes, the scenario was targeted at assessing the manual flying skills as these were suspected to be the critical point in comparable situations. While the EBT data analysis showed that manual flying skills are an issue, the first Man4Gen experiment did not show any problems, according to flight instructor ratings, in manual flying skills. However, the analysis of the first set of experiments showed that the identified problems lay within situation awareness and rushed decisions, the latter was clearly induced by the time critical scenario (dual engine bird strike in ground proximity). Therefore, the scenario of the second simulator study, described in this paper, was focused on decision-making, situational awareness and system knowledge in a situation that starts harmless but slowly deteriorates while options are running out. The task was to assess the decision-making process instead of pushing the crew to rushed decisions. Even more, the scenario was designed to be diffuse and without a clear-cut solution. This second scenario should also create the appropriate environment for evaluating a newly designed display (Risk Information Display) [5] and procedure (Complex Situation Management Guide) [6] to support crews in such situations. As the new display was designed to provide additional information for the new procedure, experiments at DLR included one group of crews that used the new display as well as the new procedure. Furthermore there was one group solely using the new procedure which performed the simulator runs at the NLR. Finally, a baseline group took part in the experiments at DLR and NLR that was not provided with any modification in the cockpit and the procedures.

In order to compare the results, a scenario had to be designed that was applicable to DLR's AVES A320 research simulator as well as NLR's GRACE A330 research simulator. By combining results from the two research sites the participant sample was increased and the effects of the modifications were analyzed between the three groups mentioned above. Based on the results of the first exploratory experiment and considering the characteristics of the two different simulators the following scenario design constraints were defined:

\section{Unexpected, ambiguous situation with focus on decision-making}

- The resulting situation shall be unexpected for the crew.

- The combination of decision events shall not correspond to existing training/procedures.

- Elements shall require decision-making by the crew but shall be benign enough not to cause an emergency landing.

- The combination of elements shall create a diffuse, ambiguous and complex situation that has no clear-cut solution.

\section{Operationally realistic}

- Elements shall be within the realm of the operationally possible, but not necessarily highly likely from a systems perspective.

- The simulation environment and the flight deck behavior shall be representative for the type of aircraft being simulated.

\section{Transferability between different aircraft types}

- The challenges imposed on the crew by each scenario element shall be comparable for Airbus A320 and A330.

\section{B. Simulator Scenario General Overview}

The scenario was designed as a commercial line flight departing from the home base of the corresponding airline. For the Airbus A320 simulation the briefing was provided for a flight between Frankfurt Airport (Germany) and Bastia-Poretta Airport (Corsica, France). In case of the Airbus A330 simulation the flight was planned between Amsterdam Schiphol (The Netherlands) and Entebbe (Uganda).

The scenario was set up with the aircraft being positioned at the departure runway's holding point when the crew entered the cockpit. After the departure briefing the crew departed and followed along the pre-programmed FMS track southward, which passes just east of a squall line heading toward Frankfurt or Amsterdam, respectively. Approximately nine minutes after departure the oil temperature of engine 2 caused an ECAM advisory (flashing oil temperature indication). If the crew did not perform any countermeasures within two minutes of time, the still increasing oil temperature caused an "Engine 2 Oil Hi Temp" ECAM warning that demands for an engine 2 shut down. Shortly after the warning two additional failures are introduced by a lightning strike: a "Thrust Lever 1 Fault" and a failure that 
causes the autothrust to work unreliably. Note that the autothrust failure is not documented in the aircraft manuals. It was artificially created by the scenario designers.

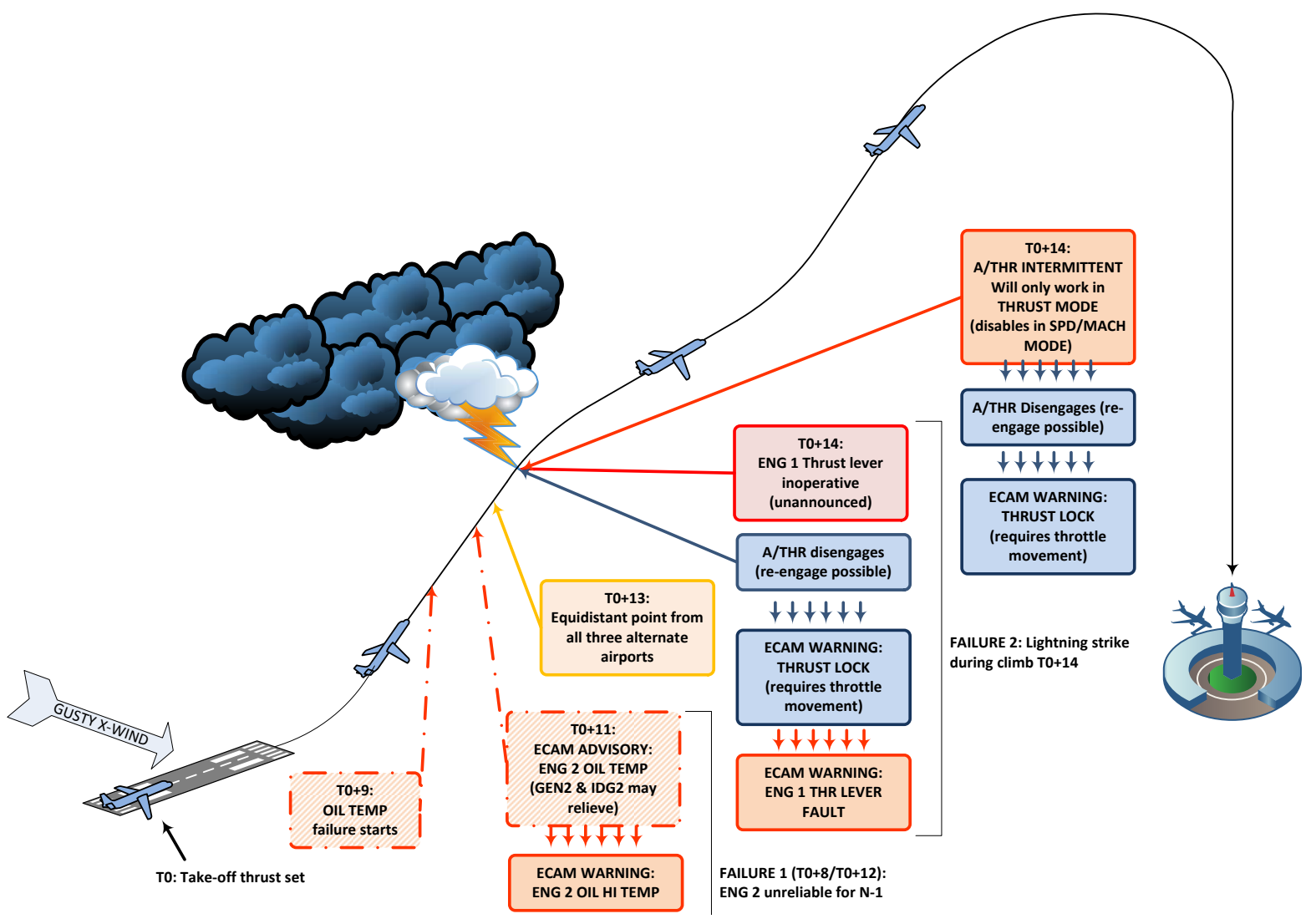

Fig. 2 Scenario overview

\section{Scenario Element Definition}

According to the challenge-centric approach, instead of defining specific system failures as starting point of the scenario design process the challenges that should be imposed on the crew were taken as basis to define each scenario element. Each scenario element and its timing is described in this section. The connection between the scenario elements and the challenges imposed on the crew are presented in the next section. The scenario elements are listed in Table 1.

\section{Engine 2 Oil High Temperature Advisory}

Approximately nine minutes after departure (cf. Table 2). the oil temperature of engine 2 starts to rise until causing an ECAM advisory (flashing oil temperature indication on the ECAM). There are several ways to reduce the engine's oil temperature according to the aircraft's Flight Crew Operating Manual (FCOM) and Quick Reference Handbook (QRH). Additionally, the increased oil temperature demands a reduced thrust level on engine 1 to keep the temperature in the limits. This event creates uncertainty for the crew concerning the status of engine 1 . The crew has to consider a reduced reliability and thus a sudden engine failure on the corresponding engine.

\section{Engine 2 Oil High Temperature Warning}

If the crew does not detect the advisory or does not react to it appropriately, the still increasing oil temperature triggers the ECAM warning "ENG 2 Oil Hi Temp" when the temperature reaches a certain level. The oil temperature rise is defined such that the ECAM warning appears two minutes after the advisory showed up (cf. Table 2). The warning demands for a shutdown of engine 2 to prevent damage to the engine. This scenario event further enhances the 
Table 1 Scenario elements

\begin{tabular}{|c|l|l|}
\hline No & Event Type & Element Name \\
\hline \hline 1 & & ENG 2 Oil High Temperature Advisory \\
2 & \multirow{2}{*}{ System Failures } & ENG 2 Oil High Temperature Warning \\
3 & & ENG 1 Thrust Lever Fault \\
4 & & A/THR Failure \\
\hline 5 & & Wind at Departure Airport \\
6 & Weather Effects & Thunderstorm Movement \\
7 & & Global Weather Situation \\
\hline 8 & & Oil Temperature Issue \\
9 & Dispatcher/Maintenance & (Auto)thrust Issues \\
10 & & Diversion Call \\
\hline
\end{tabular}

uncertainty concerning the engine 2 status. In combination with the upcoming failure (cf. next subsection) this element is included to create ambiguity in the decision-making process, because it might be required to keep the engine running (or to restart it) thereby violating the ECAM procedure.

\section{Engine 1 Thrust Lever Fault \& Autothrust Failure after Lightning Strike}

Approximately two minutes after the occurrence of the warning the aircraft passed a waypoint where an additional failure was introduced by a lightning strike: a "thrust lever 1 fault". According to the FADEC system logic, in case of a thrust lever fault, MCT is commanded on the affected engine until the slats are extended. After slats extension the system commands idle. The triggering of the lightning strike was defined by a position which corresponds to an equidistant point from the most likely alternate airports including the departure airpor* (cf. Table 2). The thrust lever fault was surprising for the crew, because it is not part of the usual recurrent training. Due to this fact it could be expected during the scenario design that most of crews do not know the detailed system behavior after a thrust lever fault by heart. Therefore it was likely that information had to be retrieved from the FCOM during the flight.

In addition to the thrust lever fault an autothrust failure was triggered at the same time. With a well functioning autothrust system the impact of the thrust lever failure is negligible as long as the autothrust is activated, because it has still full control over the engine thrust. Thus the autothrust failure was necessary to increase the complexity of the scenario. The consequence of this failure was that the autothrust could only be activated in certain modes requiring constant thrust, e.g. open climb or open descent. In those cases speed control is performed by the pitch control. As soon as the autothrust was set to speed mode (i.e. thrust controlling speed) the system was deactivated or could not be engaged at all. This complex failure behavior further generated uncertainty on the system state of the A/THR.

\section{Global Weather Situation and Thunderstorm Movement}

The global weather situation including the thunderstorm movement and the wind situation at the different alternate airports was defined such that the weather was favorable at the alternate airports in comparison to the departure airport. However, the departure airport, being the home base of the airline, was favorable from an operational point of view. In the A320 as well as the A330 scenario, at the departure time a cold front with a large squall line and embedded thunderstorms headed towards the departure airport, thereby drifting into the planned departure track. Due to the approaching front the airport was expected to close in short time. In addition to that strong cross winds were expected for the landing on the departure airport. This weather situation thus created ambiguity in the decision-making process of the crew concerning the selection of an appropriate landing airport.

\section{Resulting Overall Situation}

The combination of failures puts the crew into the situation that they have engine 2 working unreliably and engine 1 working either in MCT or IDLE thrust when A/THR is not activated, which in turn is limited to THR mode. In order to

\footnotetext{
*For the A320 scenario this was the case for the IFR waypoint ANEKI (cf. Figure 3
} 


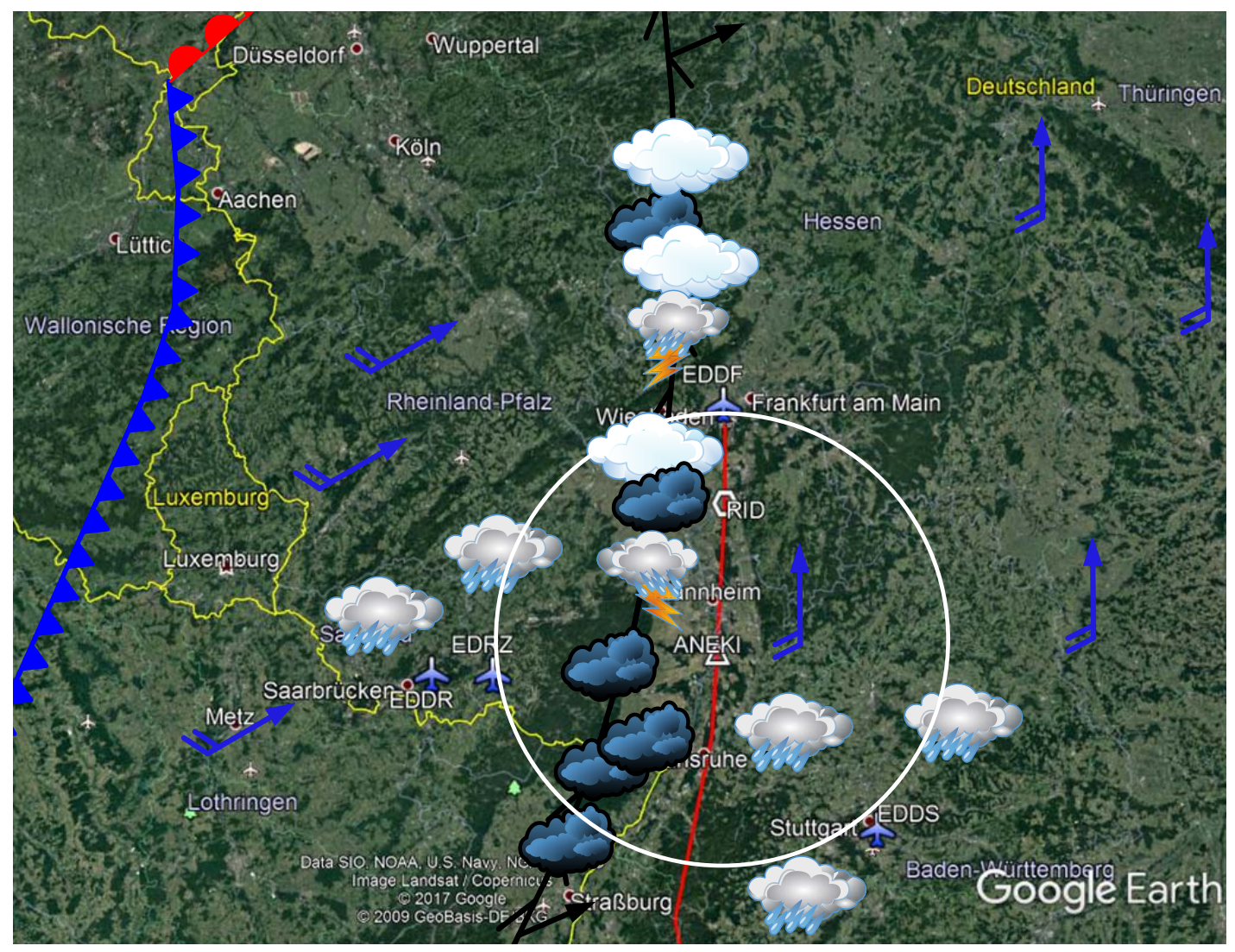

Fig. 3 Weather situation for the Airbus A320 scenario (constant distance around ANEKI in white, planned route in red) [7]

Table 2 Scenario event timing

\begin{tabular}{|c|c|c|c|}
\hline No & Scenario Event & Timing & Motivation \\
\hline 1 & $\begin{array}{l}\text { ENG } 2 \text { Oil High Temperature } \\
\text { Advisory }\end{array}$ & 9 min after $\mathrm{T} / \mathrm{O}$ & $\begin{array}{l}\text { The ECAM advisory should occur } 2 \text { min before the ECAM } \\
\text { warning. The time for countermeasures was limited in order to } \\
\text { increase the complexity and to test the system knowledge. }\end{array}$ \\
\hline 2 & $\begin{array}{l}\text { ENG } 2 \text { Oil High Temperature } \\
\text { Warning }\end{array}$ & $11 \mathrm{~min}$ after $\mathrm{T} / \mathrm{O}$ & $\begin{array}{l}\text { The ECAM warning should occur } 2 \text { min before the thrust lever } \\
\text { fault to give the crew some time to discuss the ECAM warning } \\
\text { and possible consequences. }\end{array}$ \\
\hline 2 & ENG 1 Thrust Lever Fault & $\begin{array}{l}\text { approx. } 14 \text { min after } \\
\mathrm{T} / \mathrm{O}\end{array}$ & $\begin{array}{l}\text { The thrust lever fault should occur briefly after passing a specific } \\
\text { waypoint which is equidistant to the most likely alternates. This } \\
\text { defines the approx. triggering time after T/O. }\end{array}$ \\
\hline 3 & A/THR Failure & $\begin{array}{l}\text { simultaneous with } \\
\text { thrust lever fault }\end{array}$ & $\begin{array}{l}\text { To immediately create a complex situation this failure is triggered } \\
\text { in conjunction with the thrust lever fault. Additionally, at this } \\
\text { position, due to ATC instructions, the aircraft was in a horizontal } \\
\text { flight for some time resulting in the A/THR being in speed mode. } \\
\text { This caused an immediate disengagement of the A/THR leading } \\
\text { to a surprising effect. }\end{array}$ \\
\hline
\end{tabular}

keep the oil temperature of engine 2 within the given limits thrust has to be kept close to idle.

For being able to solve the scenario the crew has to identify the aircraft status and the logic of the engine 1 thrust control. With this in mind the crew could then decide for a diversion airport and plan their descent and approach accordingly. As 
the squall line is approaching Frankfurt and would arrive there by the time the crew would return, they are challenged in their diversion decision as their preferred return field has become a less preferable option. The different system failures in conjunction with the global weather situation creates a complex and ambiguous situation. The timing of each scenario event is defined so that the situation deteriorates until the lightning strike occurs (cf. Table 2). Although the decision-making process is complex, because there is no clear-cut solution, the situation is still benign enough to provide the crew with sufficient time for the decision-making process and their long-term planning which is the focus of the scenario.

The following listing summarizes the circumstances that had to be considered by the crew during the decision-making process:

- Engine 2 thrust can only be set above idle for a limited time without reaching the oil temperature limits. There might be some doubt on the reliability of the engine, especially if the ECAM warning was triggered during the scenario.

- Engine 1 is not controllable at all. Enough thrust is available to fly in level flight. However, the overall thrust is too high to descent with an acceptable speed without using the speed brakes or the landing gear to increase drag.

- If the crew switched off engine 2 in response to the high oil temperature ECAM warning, the crew is left with one uncontrollable engine.

- For an appropriate problem solving and long-term planing, system knowledge about the FADEC behavior after a thrust lever fault is required, as it sets the thrust to idle after slat extension. With the reduced reliability of engine 2 and with engine 1 being stuck in idle a go-around is not an option. This affects the selection of an appropriate alternate airport.

- The weather situation at the departure airport is uncertain, as it might be closed due to the moving thunderstorms. However, the departure airport is the home base, which is the best alternate airport from a maintenance point of view.

- Some of the possible alternate airports have strong crosswind conditions. Considering the asymmetric thrust and the limited go-around capabilities this might reduce safety margins.

\section{Dispatcher/Maintenance Support}

In order to generate an operationally realistic scenario the crew is able to contact an airline dispatcher and maintenance support. A script for the simulated dispatch/maintenance support provided the answers to possible technical questions in order to keep all experimental runs comparable. If the crew asked questions about the preferable landing airfield, the dispatcher or maintenance support would increase the ambiguity of the scenario by stating the advantages of the home base from an operational point of view.

\section{Challenge-centric Scenario Element Definition}

The design process presented in this paper differs from conventional scenario design approaches in using the desired crew impact or challenges as basis for the scenario design. In this section the connection between the expected crew impact and each scenario element is presented. It should be noted that the scenario described in the preceding sections is just one possible solution. A similar crew impact could be realized with different scenario elements or events, which do not necessarily result exclusively from system failures but can also be caused by weather effects, by ATC communication or by other traffic. The scenario design task is complex, because for the assessment of the crew impact the scenario elements cannot be considered as isolated items. It is rather the combination of different scenario elements that creates the challenges for the crew.

\section{E. Scenario Implementation on Different Aircraft Types}

According to the scenario design constraints it was crucial that the scenario was realistic in terms of airline operations, but also in terms of aircraft system behavior. Both system failures as well as the weather effects had to be slightly adapted to the system behavior and the operational constraints of both aircraft types used for the experiments, being an Airbus A320 in case of the AVES simulator and an Airbus 330 in case of the GRACE simulator. Based on the challenge-centric design approach the adaptations were performed so that the challenges for the crew and the experimental results were as comparable as possible for both simulators. 
Table 3 Crew effect and scenario characteristics

\begin{tabular}{ll}
\hline Crew Effect & Scenario Characteristic \\
\hline \hline Ambiguous decision-making & $\begin{array}{l}\text { Return to the departure airport to facilitate maintenance vs. risk of } \\
\text { airport closure due to approaching thunderstorms } \\
\text { - Most suitable diversion airports causing longer flight time with unreli- } \\
\text { able right-hand engine and increased fuel consumption } \\
\text { - Restart/keep right-hand engine after left-hand engine thrust lever fault } \\
\text { although violating SOPs on ECAM }\end{array}$ \\
\hline Surprise & $\begin{array}{l}\text { - Thrust lever fault usually not part of standard airline training } \\
\text { Enhanced Monitoring }\end{array}$ \\
\hline Complex long-term planing & Detection of high oil temperature ECAM advisory on right-hand engine \\
\hline Consideration of reduced go-around capabilities due to FADEC behav- \\
ior after slat extension in case of thrust lever fault \\
- Consideration of strong crosswind at some of the alternate airports \\
- Consideration of thunderstorm movement to departure airport \\
cruise and descent and bothe engines almost in IDLE during approach
\end{tabular}

Uncertainty about system status / Confusion

- Reliability of right-hand engine due to increased oil temperature

- Unreliable A/THR function due to A/THR failure

Deep system knowledge

- Knowledge of the FADEC behavior after ENG 1 thrust lever failure

\section{Oil Temperature Rising Time}

Since both aircraft types have different temperature levels for the ECAM advisory and the ECAM warning the rising of the oil temperature had to be adapted in such way that the advisory and warning were triggered according to Table 2 for both simulators. The same timing of the scenario events ensured an equal impact on the crews for both simulators.

\section{Global Weather Situation}

As the departure airport was different for both simulators the global weather situation had to be adapted. In both cases thunderstorms moved to the departure airport creating uncertainty about a possible closure of the airport.

\section{F. Scenario Design Assessment}

As described in the introduction of this paper the scenario was used for simulator trials within the Man4Gen project in order to assess the benefits of the developed recommendations in the field of procedures and system design. A total number of 18 crews took part in the experiments. Each crew was composed of a Captain and a First Officer both being from the same airline. All crews reported the scenario to be challenging and diffuse and that they were not used to such a complex situation from their recurrent training.

The ambiguity and the complexity of the scenario led to extensive crew decision-making processes on the energy management strategies and the available deviation airports. Due to the ambiguity of the situation there was no preferred deviation airport. Almost all crews deviated to a different airport. Six crews even returned to their home base (Frankfurt or Amsterdam) although there was some uncertainty about a possible closure of the airport and strong cross winds on 
the available runways.

The assessment of the crew performance was carried out using the so-called Desired Flight Crew Performance (DFCP) evaluation checklist containing the expected crew actions for a certain flight event [8]. Crews would get a point every time a certain DFCP item was accomplished. The points were then summed up for each crew, meaning that flight crews with higher sums showed better performance in terms of DFCP. Some of the results of the DFCP are presented in the following paragraph to illustrate the complexity and ambiguity of the designed scenario.

Seven crews did not verbalize the rising oil temperature on engine 2 and almost no crew applied counter measures to reduce the oil temperature of engine 2 .

The majority of the crews kept the engine 2 activated or restarted it during approach and landing after the thrust lever fault on the other engine had occurred. However, two crews decided not to use engine 1 for the approach and were thus fully relying on engine 2 . Only seven crews tried to maintain engine 2 below the temperature limits.

Some differences were observed in the landing preparation between the crews. Only five crews checked the impact of the failures on landing performance, while 13 crews discussed the go-around performance risks.

Generally, the extensive problem solving and decision-making process during the scenario showed that the main scenario design tasks were fulfilled.

However, during the Man4Gen design process and scenario design assessment the following issues were observed:

- The design process was very time consuming making its application to airline flight crew training very expensive.

- Whereas the Man4Gen scenario design approach focused on crew challenges, future flight crew training, such as EBT, will focus on the design of scenarios according to required competencies. Thus the training of specific competencies defined by EBT shall serve as a basis for the development of a future scenario design kit.

Especially, the extensive effort for designing scenarios based on expected crew challenges or required competencies makes the application of such a design approach to flight crew training difficult. It is thus envisioned to automate the process of scenario design and implementation. This led to research activities aiming at the development of a future scenario toolkit with the main focus on automation. The basic features of the toolkit are presented in the next section.

\section{The Future Scenario Toolkit}

In the scope of the Man4Gen scenario design process each scenario element and the timing of the events was selected to impose certain challenges on the pilots. The design approach is similar to the design process for Evidence-based Training scenarios where the task is to design scenarios that train particular competencies. The major challenge lies in making several scenarios which all train the same competencies or challenges with different contexts; this makes such scenario design a very challenging and time consuming task for Approved Training Organizations. Accordingly DLR and NLR started a research initiative to develop dedicated tools to support training organizations in designing, implementing and performing simulator scenarios that are applied within the scope of Evidence-based Training. The automation of the scenario development in this context is described as selecting the element of a scenario from a set of pre-defined scenario elements and configuring their parameters automatically according to the intended training goals. Figure 4 illustrates the current concept of the future scenario toolkit.

\section{A. Competency-based Training Scenario Design Tool}

The competency-based training scenario design tool will support the Approved Training Organization in designing operationally relevant scenarios driven by specific training goals (cf. Figure 4). For this tool the training goals will be defined in terms of competencies that shall be trained by the scenario assuming that the focus of the session is on improving the crew's competencies rather than basic flying skills for a specific maneuver, such as maximum crosswind landings.

For that reason a competency-based scenario element library is used as part of the scenario design tool (cf. Figure 47. This library includes a database of scenario elements each related to the training of specific competencies. As a starting point the library will be based on the Evidence-based Training Programme Development Guidance tables defining different scenario elements sorted by aircraft generation and training topic [3]. These tables map each scenario element to the competencies that shall be trained. However, each scenario element has to be further defined in detail for the specific aircraft type. In addition to that the database will be regularly updated using operational flight data or accident/incident data. The formal definition of the competency-based scenario elements will provide the capability to trace them back to training goals in terms of competencies.

Two development steps are envisioned for the implementation of the scenario design tool. In a first step the tool will provide a Graphical User Interface allowing a flight instructor to perform the design of the scenario in terms of 


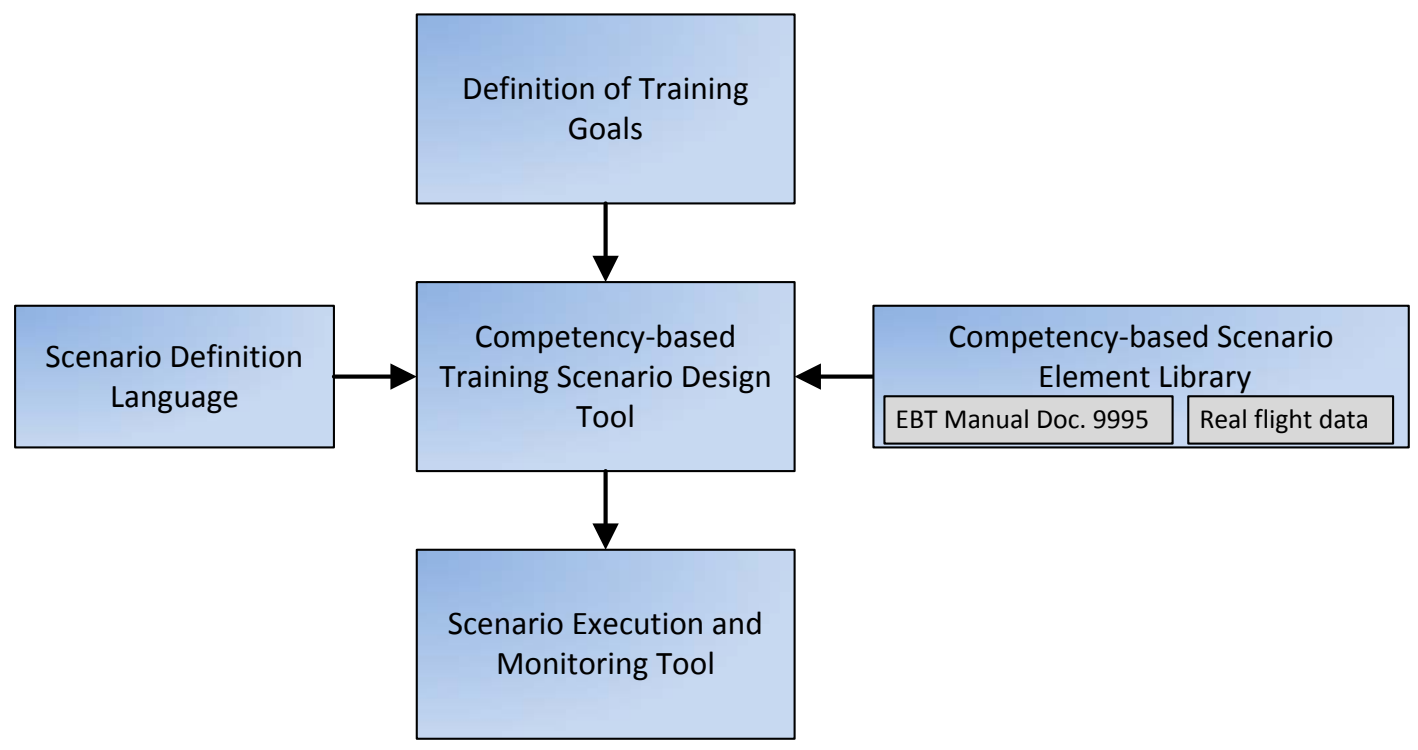

Fig. 4 Scenario toolkit overview

element selection, timing of events as well as combining different scenario elements. In this case the flight instructor will manually select the scenario elements from the library based on the competencies that shall be trained.

In a final development step the scenario toolkit shall generate training scenarios automatically based on the selected set of training goals. This will include the selection of the elements and the definition of their parameters as well as the sequencing of all events. The automatic generation of the scenarios requires a formal specification of the training goals. The challenge lies not only in formalizing the training goals but also finding the mechanism to trace them back to scenario elements, their timing and their combinations. The automated selection and sequencing of a scenario element in order to create surprise and achieve required training goals while keeping the scenario operationally realistic and flyable is regarded as a research challenge that will be tackled within this scenario toolkit research in the long run.

All scenario elements as well as the complete scenario will be formalized in a Domain Specific Language (DSL) for scenario development. While the language will capture the competency-based scenario elements, the scenario toolkit will provide necessary means to design and update the language.

A DSL is a customized computer language that provides a limited expressiveness for a particular problem domain [9]. It encompasses language features for the concepts and the constraints of the particular domain. Despite the gravity of scenarios particularly in flight training and generally in aviation has been well-known for a long time, unfortunately there is no standardized language for specifying simulation scenarios. Addressing this problem the American Institute of Aeronautics and Astronautics (AIAA) Modeling and Simulation Technical Committee (MSTC) recently launched a working group towards the development of a standard simulation scenario definition language for the aviation domain, namely AIAA Simulation Scenario Development Working Group (AIAA SSDWG). Within this context, the current practices, problems and opportunities in scenario development [10-13], the requirements of a standard simulation scenario definition language [14-16] and possible technical solutions [17--20] are currently being discussed.

Here for the scenario toolkit, we would like to exploit the outcomes of the standardization effort from AIAA SSDWG. Scenario development is a data engineering activity in which the data elements of a scenario are modeled. Following the simulation data modeling approach proposed by Zeigler and Hammonds [21], Durak et al. [19] proposed a scenario definition language based on System Entity Structure (SES). SES can be introduced as an high-level ontology for knowledge representation of decomposition, taxonomy and coupling of systems [22]. The proposed simulation scenario definition language uses decomposition, taxonomy and coupling constructs of SES for creating a formal specification of simulation scenario elements. Durak and colleagues later proposed an XML based computational representation of their simulation scenario definition language [20]. An XML Schema that extends the SES ontology is used to specify the scenario definition language. A representative excerpt from this schema is depicted in Figure 5. The elements of scenarios that are captured in the schema include Environment, Entities such as the Aircraft with its systems and Airport and Events such as system failures or weather changes. Attributes are then used to define the scenario parameters. 
Guards are used to specify the circumstances that the Events are fired and Actions are used to specify which attributes are changed as a consequence of the fired event.

The XML schema of SES is then used to define the Simulation Scenario Definition Language as an XML, which is eventually converted to the Simulation Scenario Definition Language XML Schema. The elements of the XML schema of the language, namely the entities, attributes, sequences or choices capture all the possible scenario elements, their relations and constraints. Then, any valid instance of this schema is a simulation language. SES XML Schema validates the well-formedness of the language and XML Schema of the language guaranties the well-formedness of a scenario. The details of the approach are presented in Durak et al.[20].

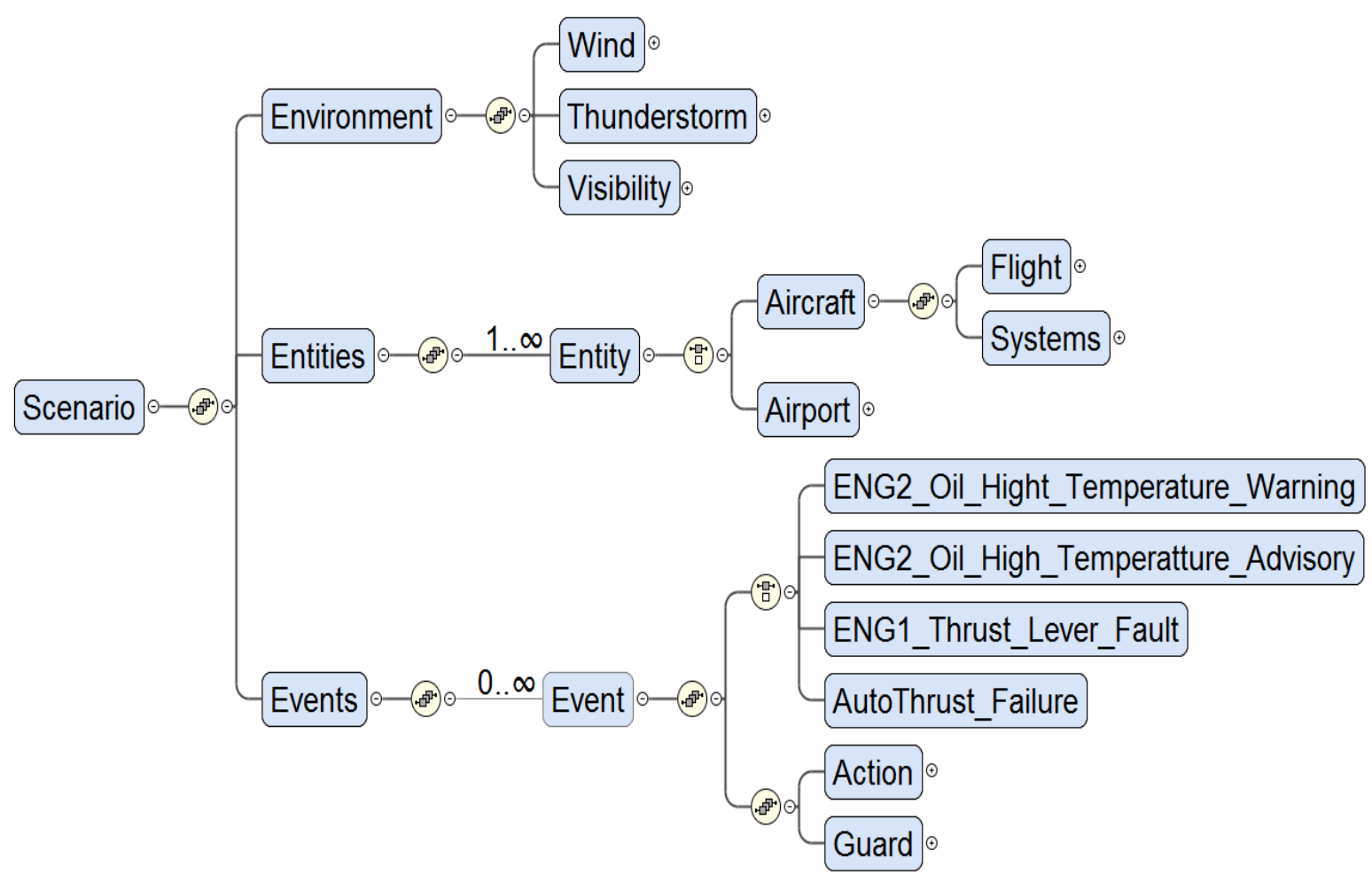

Fig. 5 An excerpt from the simulation scenario definition XML schema

\section{B. Scenario Execution and Monitoring Tool}

As presented in Figure 5 the Event path contains Guard and Action nodes. The Guard defines the event triggering conditions, whereas the Action node contains the values or states that have to be changed. This allows an automatic triggering of each scenario event resulting in an identical sequencing of the events of each scenario run.

The last step of the scenario development that will be carried out by the scenario toolkit is the distribution of the scenario to the simulation applications, inject the events during the simulation execution and monitor the performance measures related to the training goals. It requires a standardized simulator interface that is capable of importing the XML Schema that is created by the competency-based training scenario design tool. This is regarded rather a straight forward scenario management task.

\section{Conclusion}

This paper presents the challenge-centric scenario design approach applied to the Man4Gen project. The challengecentric design approach focuses on the impact of each scenario element on the crew. Using the Man4Gen scenario as example each scenario element and its connection to the expected crew impact or crew challenge is described in detail. It is shown that the crew challenges are not only generated by system failures but also by the global weather situation, by ATC or by the airline dispatch and maintenance center. 
A specific requirement of the Man4Gen scenario design process was the application of the scenario to two different aircraft types and research simulators, being the DLR AVES simulator in Braunschweig and the NLR GRACE simulator in Amsterdam. This design constraint further forced the scenario designers to focus on the crew impact. Particular system failures were designed so that comparable crew impacts were provoked in the DLR Airbus A320 as well as in the NLR Airbus A330 simulator.

However, the Man4Gen example showed that such a design approach can be very time consuming and thus might be too expensive for airline training organizations. As at the same time Evidence-based Training is getting more and more promoted by ICAO, regulators and airline industry, it is recommended to define the expected impact on the crew in terms of required competencies than in terms of challenges.

In summary this led to the initiation of research activities concentrating on the development of a toolkit for the design, the implementation and the conduction of training scenarios supporting the training of specific competencies as defined in the EBT training manual [3].

The scenario kit will be composed of two different software tools: the competency-based training scenario design tool and the scenario execution and monitoring tool. The scenario design tool shall support the ATOs in defining operationally relevant scenarios. In the first step it is planned to provide the scenario designers with a graphical user interface allowing the definition of the scenario using a competency-based scenario element library. Each scenario element shall be assigned to one or more competencies according to [3]. In a further future step it might be possible to develop algorithms that even allow the automatic scenario design according to the training task (i.e. the training of specific competencies) defined by the flight instructor. As part of this scenario design tool a Domain Specific Language for scenario development is envisioned as the underlying infrastructure.

Then, an automatic processing of the graphically designed scenario shall lead to an XML Schema which shall be serve as the input to the simulator operator station. The scenario execution and monitoring tool takes care of the execution of the designed scenario by triggering the events and setting the according parameter values during the simulation. Therefore this toolkit will guarantee a consistent process from the design to the conduction of a scenario within a limited time frame.

\section{Acknowledgments}

The authors would like to thank the flight crews that participated in the Man4Gen project, as well as the experts and other consortium partners that contributed to the experiment work at NLR and DLR. The Man4Gen research is funded as part of the FP7 2012 Aeronautics and Air Transport programme under EC contract ACP2-GA-2012-314765-Man4Gen. The views and opinions expressed in this paper are those of the authors and do not necessarily represent the position and opinions of the Man4Gen consortium and/or any of the individual partner organizations. If you have any questions regarding the Man4Gen project, please contact man4gen@nlr.nl.

\section{References}

[1] Australian Transport Safety Bureau, "In-flight Uncontained Engine Failure, 4 November 2010, Airbus A380-842, VH-OQA," Tech. Rep. AO-2010-089, Australian Transport Safety Bureau, 2013. URLhttps://www . atsb.gov . au/media/4173625/ao2010-089_final.pdf

[2] Komite Nasional Keselamatan Transportasi, "Aircraft Accident Investigation Report, PT. Indonesia Air Asia, Airbus A320-216; PK-AXC," Tech. Rep. KNKT.14.12.29.04, Komite Nasional Keselamatan Transportasi, 2014. URL https://reports. aviation-safety.net/2014/20141228-0_A320_PK-AXC.pdf

[3] International Civil Aviation Organization, "Manual of Evidence-based Training - ICAO Doc 9995," Tech. rep., International Civil Aviation Organization, 2013. URL https://www . icao .int/SAM/Documents/2014-AQP/EBT\%20ICA0\%20Manual\% 20Doc\%209995.en.pdf

[4] International Air Transport Association, "Data Report for Evidence-Based Training," Tech. rep., International Air Transport Association, 2014. URL https://www.iata.org/whatwedo/ops-infra/training-licensing/Documents/datareport-for-evidence-basted-training-aug\%202014.pdf

[5] Buch, J.-P., Niedermeier, D., and Stepniczka, I., "Managing the Unexpected - Human-in-the-Loop Simulation as Effective Tool for the Assessment of the Risk Information System in an Operationally Relevant Context," AIAA Modeling and Simulation Technologies Conference, AIAA AVIATION Forum, American Institute of Aeronautics and Astronautics, 2017. doi:10.2514/6.2017-4155, URL https://doi.org/10.2514/6.2017-4155 
[6] Field, J., Lemmers, A., and Boland, E., "Are Pilots in Control? How do pilots react to unexpected situations?" AIAA Modeling and Simulation Technologies Conference, AIAA SciTech Forum, American Institute of Aeronautics and Astronautics, 2016. doi:10.2514/6.2016-1432, URL https://doi.org/10.2514/6.2016-1432

[7] Google Inc., "Google Earth Pro Version 7.3.0.3832 (32-bit) [Computer Software],", Aug 2017. URL https://www.google. de/earth/.

[8] Fucke, L., "D6.4 - Final Report of Operational Recommendations," Tech. Rep. 314765 Man4Gen - Public Deliverable, European Union, 2016.

[9] Fowler, M., Domain-specific Languages, Pearson Education, 2010.

[10] Reardon, S., Lewis, E., and Beard, S. D., "Scenario Development Process at the Vertical Motion Simulator," AIAA Modeling and Simulation Technologies Conference, Grapevine, TX, 2017.

[11] Suikat, R., Stelkens-Kobsch, T., Stiekel, F., and Lenz, H., "Creating Scenarios for Airport Management Simulations Involving Independent Connected Simulators," AIAA Modeling and Simulation Technologies Conference, Grapevine, TX, 2017.

[12] Crider, D. A., "The Use of Data from Accident Investigations in Development of Simulator Training Scenarios," AIAA Modeling and Simulation Technologies Conference, Grapevine, TX, 2017.

[13] Rigby, K. T., Macchiarella, N. D., and Mirot, A., "Enhanced Scenario-Based Training for Unmanned Aircraft System Operational Missions," AIAA Modeling and Simulation Technologies Conference, Grapevine, TX, 2017.

[14] Field, J., Gerretsen, A., Meiland, R., and Mohrmann, F., "Harmonizing the Scenario Generation Process, Tools and Standards for Flight Simulators," AIAA Modeling and Simulation Technologies Conference, Grapevine, TX, 2017.

[15] Durak, U., Jafer, S., Beard, S. D., Reardon, S., Murphy, J. R., Crider, D. A., Gerretsen, A., Lenz, H., Macchiarella, N. D., Rigby, K. T., Torens, C., and Adolf, F.-M., "Towards a Standardization for Simulation Scenario Development in Aviation - Panel Discussion," AIAA Modeling and Simulation Technologies Conference, Kissimmee, FL, 2018.

[16] Jafer, S., and Durak, U., "Tackling the complexity of simulation scenario development in aviation," Proceedings of the Symposium on Modeling and Simulation of Complexity in Intelligent, Adaptive and Autonomous Systems, Society for Computer Simulation International, 2017, p. 4.

[17] Jafer, S., Chhaya, B., Durak, U., and Gerlach, T., "Formal Scenario Definition Language for Aviation: Aircraft Landing Case Study," AIAA Modeling and Simulation Technologies Conference, Washington, DC, 2016. doi:10.2514/6.2016-3521, URL https://doi.org/10.2514/6.2016-3521.

[18] Jafer, S., Chhaya, B., and Durak, U., "Graphical Specification of Flight Scenarios with Aviation Scenario Defintion Language (ASDL)," AIAA Modeling and Simulation Technologies Conference, Grapevine, TX, 2017.

[19] Durak, U., Pruter, I., Gerlach, T., Jafer, S., Pawletta, T., and Hartmann, S., "Using System Entity Structures to Model the Elements of a Scenario in a Research Flight Simulator," AIAA Modeling and Simulation Technologies Conference, Grapevine, TX, 2017.

[20] Durak, U., Jafer, S., Wittman, R., Mittal, S., Hartmann, S., and Zeigler, B. P., "Computational Representation for a Simulation Scenario Definition Language," AIAA Modeling and Simulation Technologies Conference, Kissimmee, FL, 2018.

[21] Zeigler, B. P., and Hammonds, P. E., Modeling and simulation-based data engineering: introducing pragmatics into ontologies for net-centric information exchange, Academic Press, 2007.

[22] Kim, T. G., Lee, C., Christensen, E. R., and Zeigler, B. P., "System Entity Structuring and Model Base Management," IEEE Transactions on Systems, Man, and Cybernetics, Vol. 20, No. 5, 1990, pp. 1013-1024. doi:10.1109/21.59966. 